\title{
Treatment with Convalescent Plasma for Influenza A (H5N1) Infection
}

TO THE EDITOR: A previously healthy 31-year-old male van driver presented to a local clinic in Shenzhen, in southern China, on June 7, 2006, with a 4-day history of a high fever (temperature, $39.9^{\circ} \mathrm{C}$ ), chills, and a cough with clear sputum. A chest radiograph obtained on June 9 revealed large opacities in the lower lobe of the left lung. A reversetranscriptase-polymerase-chain-reaction (RT-PCR) assay and an isolate from a tracheal aspirate were positive for influenza A (H5N1) virus. Treatment with $150 \mathrm{mg}$ of oseltamivir twice daily was started at 2 a.m. on June 12 (Fig. 1). After 2 days, there was increased consolidation in the right lung and only slight improvement in the left lung. Quantitative RT-PCR revealed a high viral load $\left(1.31 \times 10^{5}\right.$ copies per milliliter) when it was first measured (at 4 a.m. on June 13), 26 hours after the patient began to receive oseltamivir. The viral load continued to increase and was $1.68 \times 10^{5}$ copies per milliliter by 10 a.m. on June 14, despite the continuation of oseltamivir treatment. Although oseltamivir resistance during the treatment of influenza A (H5N1) virus infection has been reported, ${ }^{1}$ no genetic evidence of resistance to oseltamivir was observed in our patient.

Because a predominant $\mathrm{H} 5 \mathrm{~N} 1$ influenza variant (clade 2.3) had been identified in poultry across southern China, ${ }^{2}$ convalescent plasma was obtained from a patient from Anhui province who had recovered from H5N1 infection in February 2006. Three 200-ml transfusions of convalescent plasma (neutralizing antibody titer, 1:80 dilution)

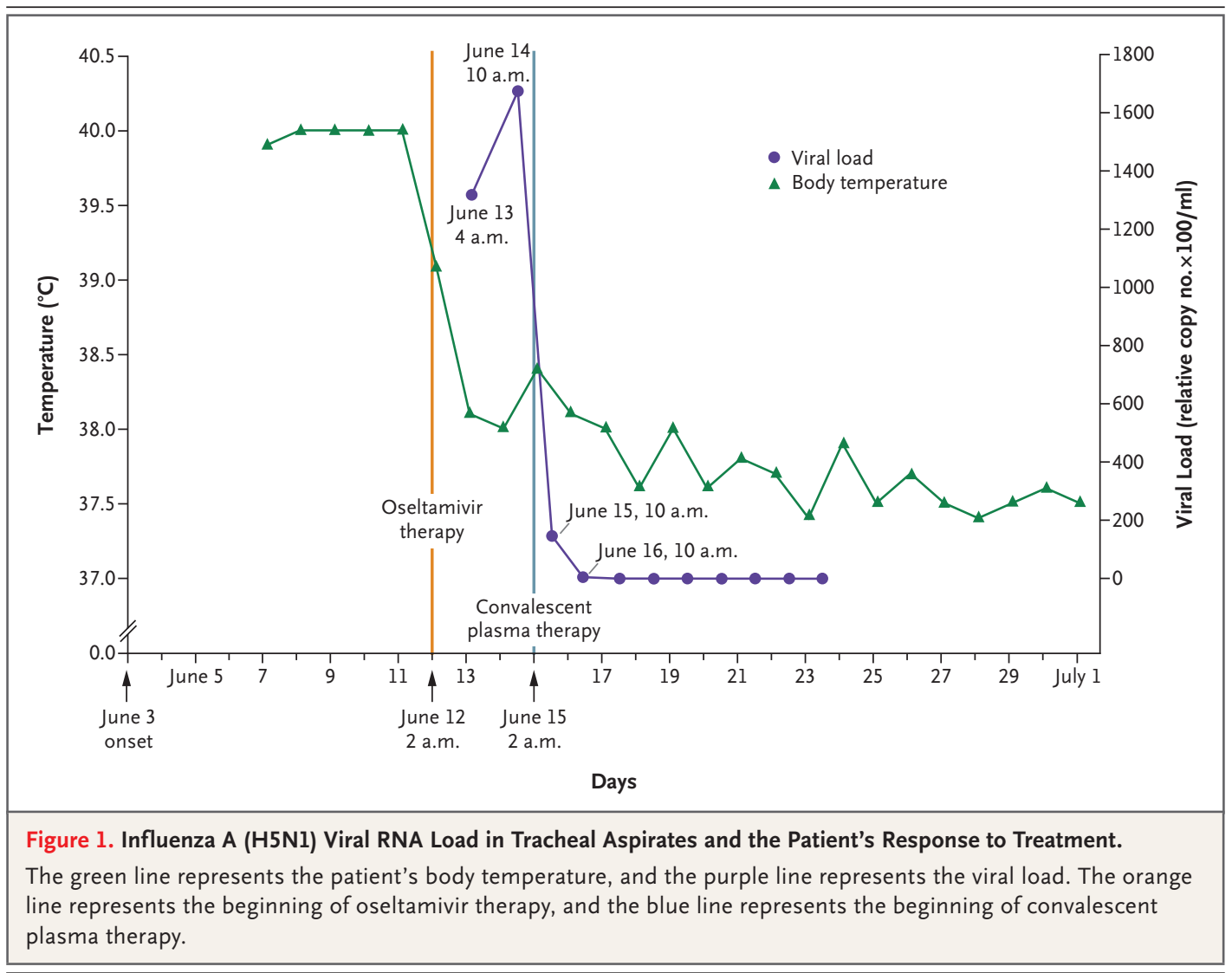


were given to our patient; one was given at 2 a.m. on June 15, one at 10 a.m. on June 15, and one at 10 a.m. on June 16. After the first transfusion, the patient's viral load was reduced by a factor of approximately 12 (from $1.68 \times 10^{5}$ to $1.42 \times 10^{4}$ copies per milliliter) during the first 8 hours (from 2 a.m. to $10 \mathrm{a} . \mathrm{m}$. on June 15) and was undetectable within 32 hours. A radiograph obtained on June 15 showed reduced density of the pulmonary lesions in the left lobe; however, consolidation in the right lower lobe had progressed. Oseltamivir treatment was stopped at 10 a.m. on June 16 because of persistently negative results on RT-PCR. The patient recovered and was discharged on $\mathrm{Au}-$ gust 4.

We performed tests of neutralizing antibodies against A/chicken/Hong Kong/282/2006, a virus closely related to A/Shenzhen/406H/2006. The neutralizing-antibody titer was negative on June 14 and 15, then it rose steadily and was between 1:40 and 1:80 by June 20 . This increase may have been the result of both the treatment with convalescent plasma and the patient's own humoral immune response, since the neutralization antibodies were maintained at this level thereafter. H5N1 viruses were successfully isolated from tracheal aspirates from both this patient and the plasma donor. A subsequent analysis revealed that the virus from the plasma donor and the current patient were closely related genetically, with greater than $99 \%$ homology in their hemagglutinin genes. Both viruses were Fujian-like H5N1 variants that have been predominant in poultry and have caused other human infections in southern China since 2005 (e.g., A/Guangxi/1/2005, A/Zhejiang/16/2006, and A/chicken/Hong Kong/282/2006). ${ }^{2}$ Our results indicate that passive immunotherapy may be a viable option for the treatment of influenza $\mathrm{A}$ (H5N1) infection. Therefore, the development of passive immunotherapy with humanized monoclonal or polyclonal antibodies warrants further consideration. ${ }^{3-5}$

\section{Boping Zhou, M.D., Ph.D.}

Shenzhen Donghu Hospital

Shenzhen 518020, China

Nanshan Zhong, M.D.

Guangzhou Institute of Respiratory Disease

Guangzhou 510120, China

Yi Guan, M.D., Ph.D.

University of Hong Kong Hong Kong SAR, China

yguan@hkucc.hku.hk

1. de Jong MD, Tran TT, Truong HK, et al. Oseltamivir resistance during treatment of influenza A (H5N1) infection. $\mathrm{N}$ Engl J Med 2005;353:2667-72.

2. Smith GJD, Fan XH, Wang J, et al. Emergence and predominance of an H5N1 influenza variant in China. Proc Natl Acad Sci U S A 2006;103:16936-41.

3. Luke TC, Kilbane EM, Jackson JL, Hoffman SL. Meta-analysis: convalescent blood products for Spanish influenza pneumonia: a future H5N1 treatment? Ann Intern Med 2006;145:599609.

4. Hanson BJ, Boon ACM, Lim APC, Webb A, Ooi EE, Webby RJ. Passive immunoprophylaxis and therapy with humanized monoclonal antibody specific for influenza A $\mathrm{H} 5$ hemagglutinin in mice. Respir Res 2006;7:126.

5. Lu J, Guo Z, Pan X, et al. Passive immunotherapy for influenza A H5N1 virus infection with equine hyperimmune globulin $\mathrm{F}\left(\mathrm{ab}^{\prime}\right) 2$ in mice. Respir Res 2006;7:43.

\section{Tissue-Engineered Blood Vessel for Adult Arterial Revascularization}

TO THE EDITOR: A material that will approach the efficacy of native vein has been widely sought. ${ }^{1}$ Using autologous cells and a technique termed sheet-based tissue engineering, we were able to produce autologous tissue-engineered blood vessels with physiologic mechanical properties. ${ }^{2}$ No synthetic or exogenous materials were used; instead, the vessels were created with the use of autologous fibroblasts and endothelial cells harvested from a small biopsy specimen of skin and superficial vein. Here we report on the preliminary use of these tissue-engineered blood vessels in an adult arterial model.

Ten patients receiving hemodialysis whose arteriovenous shunts were failing were enrolled in this study. The subjects had typical risk factors for end-stage renal disease, including previously failed dialysis-access grafts, diabetes, controlled hyper- 